\title{
Preparation and Characterization of High Magnetic Loss Microwave Absorber for Splitter Load
}

\author{
Lan Tian ${ }^{1 *}$, Dong Lichao ${ }^{1}$, Li Nan ${ }^{1}$, Zhang Chunbo ${ }^{1}$, Zhang $\mathrm{Hao}^{1}$, Pei Yuchen ${ }^{1}$ \\ ${ }^{1}$ Aerospace Institute of Advanced Materials \& Processing Technology, 100081, Beijing, CHINA
}

\begin{abstract}
Microwave absorbing material was prepared using epoxy resin as matrix and spherical carbonyl iron powder (s-CIP), flaky carbonyl iron powder (f-CIP) as absorbing agent. Microwave absorbing, mechanical properties and structure of the composites containing different kinds of carbonyl iron powder (CIP) were investigated. The results show that the microwave absorber with flaky CIP have better electromagnetic properties than composites with spherical CIP. With concentration of spherical CIP increasing the electromagnetic properties of composites becomes better, except for the mechanical performance. The flaky CIP/epoxy resins composites with a loading of 79 wt $\%$ plate-like CIP has attenuation constant of $25.443 \mathrm{~dB} / \mathrm{cm}$ in $3 \mathrm{GHz}$. The absorber prepared using flaky CIP has homogenous, dense structure and has excellent mechanical properties.
\end{abstract}

\section{Introduction}

Splitter is a kind of device that can separate multiple signals into a single frequency band. It is an indispensable component in all kinds of microwave subsystem $^{[1,2]}$. The absorption load is used to absorb the excess microwave and stabilize the system signals, especially in S-band. It is a key component of the splitter. Performances of the splitter, such as work frequency, bandwidth, return loss, insertion loss and power capacity etc, are depended on the matching load. Carbonyl iron powder (CIP), as a representative of excellent performance absorbing agent, has high magnetic permeability, good temperature stability and its industrial production technology is mature. It is one of the absorbing material which have been got the most research $^{[3-5]}$. However, the absorbing properties of spherical carbonyl iron powder (s-CIP) in low-frequency S-band still need to be improved. Here, in order to overcome the drawbacks of s-CIP, we prepare flake-like CIP/epoxy resins composites which have excellent electromagnetic properties. It is supposed that the morphology and structure have a significant impact on the absorbing properties of materials, especially for the splitter load.

\subsection{Materials}

The spherical carbonyl iron powder was purchased from Jiangxi Yuean advanced materials co. The flaky carbonyl iron powder was milled by our laboratory. Epoxy resin was provided by Jiangsu Bluestar Epoxy company(Wu $\mathrm{Xi}$ ). The curing agent was synthesized by our laboratory. Dibutyl phthalate (DBP) and acetone were supplied by Beijing chemical works (Bei Jing).

\subsection{Preparation}

The epoxy resin and curing agent were stirred with dibutyl phthalate/acetone mixture to form a homogeneous solution by a $1 \mathrm{~h}$ mechanical stirring at room temperature. Then different weights of s-CIP (80wt\%, 89wt\%) and p-CIP $(79 \mathrm{wt} \%)$ were placed into the epoxy solution, respectively and stirred thoroughly. The mixture were piled into a metal plate which had been coated releasing agent. After that, the metal plate was placed into the autoclave and the samples were cured according to the following schedule: $25{ }^{\circ} \mathrm{C}$ for $6 \mathrm{~h}, 45^{\circ} \mathrm{C}$ for $4 \mathrm{~h}, 60{ }^{\circ} \mathrm{C}$ for $4 \mathrm{~h}, 100{ }^{\circ} \mathrm{C}$ for $2 \mathrm{~h}$ at $850 \mathrm{kPa}$ as the applied pressure. Table 1 lists the mass ratio between CIP and epoxy resin.

\section{Experimental}

Table 1. The electromagnetic composites with different mass ratio between CIP and epoxy resin.

\begin{tabular}{ccccc}
\hline Sample & s-CIP/g & p-CIP/g & Epoxy resin/g & Total weight/g \\
\hline Q-1 & 800 & $/$ & 200 & 1000 \\
Q-2 & 890 & $/$ & 110 & 1000 \\
P-3 & $/$ & 790 & 210 & 1000 \\
\hline
\end{tabular}

*Corresponding author: lantiansky2016@163.com 


\subsection{Characterization}

The morphologies of the samples were characterized by scanning electron microscopy (FE-SEM S-4800, Hitach). The complex permittivity and permeability of the samples were measured by a microwave vector network analyzer (CETC-41, AV3672B-S) in the frequency range 2.6 3.95 GHz by using wave-guide reflection/transmission technique (where the E', E' and U', U" were measured). The attenuation constant of samples were calculated by such equations: attenuation constant

$(\mathrm{dB} / \mathrm{cm})=[2 \pi(8.686) / \lambda] \times[(\mathrm{E} \times \mathrm{U}) / 2]^{1 / 2} \times\left\{\left[\left(1+\tan ^{2} \delta_{\mathrm{d}}\right)\left(1+\tan ^{2}\right.\right.\right.$ $\left.\left.\left.\delta_{\mathrm{m}}\right)\right]^{1 / 2}-\left(1-\tan \delta_{\mathrm{d}} \tan \delta_{\mathrm{m}}\right)\right\}^{1 / 2}$, dielectric loss: $\tan \delta_{\mathrm{d}}=\mathrm{E} " / \mathrm{E}$, magnetic loss: $\tan \delta_{\mathrm{m}}=\mathrm{U}$ '/U'. The tensile properties of the composites were determined using dumbbell specimens with size $75 \mathrm{~mm}$ in length, $10 \mathrm{~mm}$ in width and $2 \mathrm{~mm}$ in thickness at $1 \mathrm{~mm} / \mathrm{min}$ crosshead speed on Instron 5569 instruments, USA. The flexural properties of the composites were determined using rectangular specimens with size $80 \mathrm{~mm}$ in length, $10 \mathrm{~mm}$ in width and $4 \mathrm{~mm}$ in thickness in the three point bending mode.

\section{Results and discussion}

\subsection{Morphology of CIP/epoxy resins composites}

Three kinds of CIP/epoxy resins composites are prepared and their cross-section structures are shown in Figure.1. It could be found that the Q1 and Q2 exhibit a clean and smooth spherical structure as well as has a mean particle size of approximately 4-5 $\mu \mathrm{m}$. However, Fig. 1(2) shows that the agglomeration appears in the structure of Q2 when the content of s-CIP reach to $89 \mathrm{wt} \%$. On the other hand, it can be seen that the plate-like CIP is uniformly coated with a layer of epoxy resin in Fig. 1(3). Herein, epoxy resin functioned as a binder and dispersing agent, which could not only archive a good bonding for each of $\mathrm{p}$-CIP but also disperse $\mathrm{p}$-CIP during dipping.

\subsection{Electromagnetic properties of CIP/epoxy resins composites}

Fig. 2 (1) shows the real part (E') and the imaginary part (E") of the relative complex permittivity for Q1, Q2 and P3 samples in 2.6-3.95 GHz. It can be seen that the real part E' of Q1, Q2 and P3 show some small variations in S-band. The imaginary part E" of Q1 is around 1.5 in the 2.6-3.95 GHz range. E" of Q2 and $\mathrm{P} 3$ are much smaller and is almost constant $(E "=0.5)$ in the whole frequency band. Clearly, both E' and E" of Q1 are lower than those of Q2 and P3 in the S-band. What's more, Table.1 shows the calculated dielectric loss of Q1, Q2 and P3 samples in $3 \mathrm{GHz}$ are $0.088,0.012,0.018$, respectively. This observation could be ascribed to the fact that the conglomerated plate-like structures of CIP, which will result in lower dielectric loss. It is believed that proper dielectric loss are favorable for enhancing the microwave-absorption properties in $2.6-3.95 \mathrm{GHz}$.

The permeability dispersion spectra for the three samples are shown in Fig. 2 (2). In the $2.6-3.95 \mathrm{GHz}$ range, the real part (U') of $\mathrm{P} 3$ exhibits an abrupt decrease from 4.9 to 3.4 with increasing frequency, while U' of Q2 declines slowly from 3.7 to 3.1. The U' of Q1 shows no obvious changes in the whole frequency range, indicating weak magnetic loss for Q1. The magnetic loss (U"/U') of P3 appears a resonant peak centered around $3.8 \mathrm{GHz}$. Moreover, the magnetic loss peaks around $3 \mathrm{GHz}$ for $\mathrm{P} 3$ comes from the natural resonance, which is the main magnetic loss in P3. It can be seen that P3 exhibits the strongest magnetic loss for the $3 \mathrm{GHz}$, while the dielectric loss factor of P3 is nearly zero. Obviously, the main contribution for the EM wave absorption should come from the magnetic loss for P3. What's more, as shown in Table.2, P3 also has the biggest attenuation constant in $3 \mathrm{GHz}$. This is reasonable according to the above definition of attenuation constant, higher values of U" result in higher attenuation constant.

Mechanical properties of Q1 was measured and compared with Q2 as shown in Table. 2. Compare to the $80 \mathrm{wt} \%$ s-CIP content of composite (Q1), the tensile strength (51.39 MPa) and flexural strength (93.3MPa) approximately decreased by $14.3 \%$ and $9.9 \%$, respectively, when the content of s-CIP reach the $89 \mathrm{wt} \%$. However, the P3 samples show the excellent mechanical performance, which included tensile strength (65.42MPa) and flexural strength (118.4MPa).

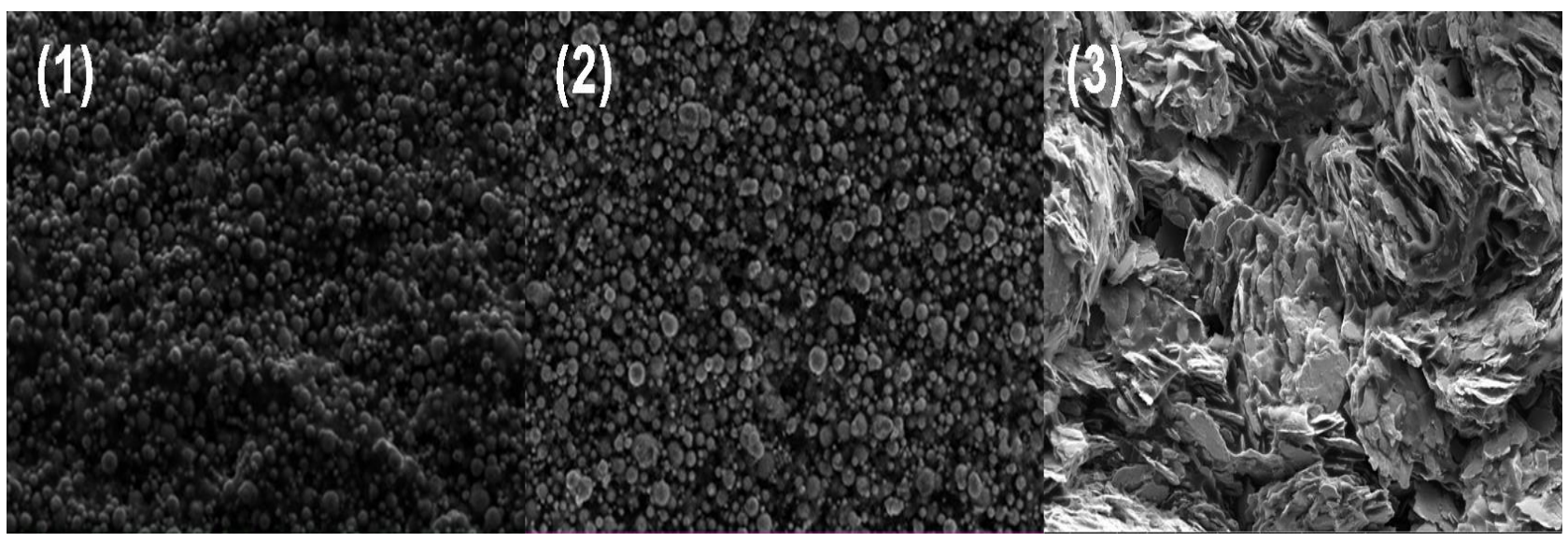

Fig.1. The cross-section structures of composites (1) Q1, (2) Q2, (3) P3. 

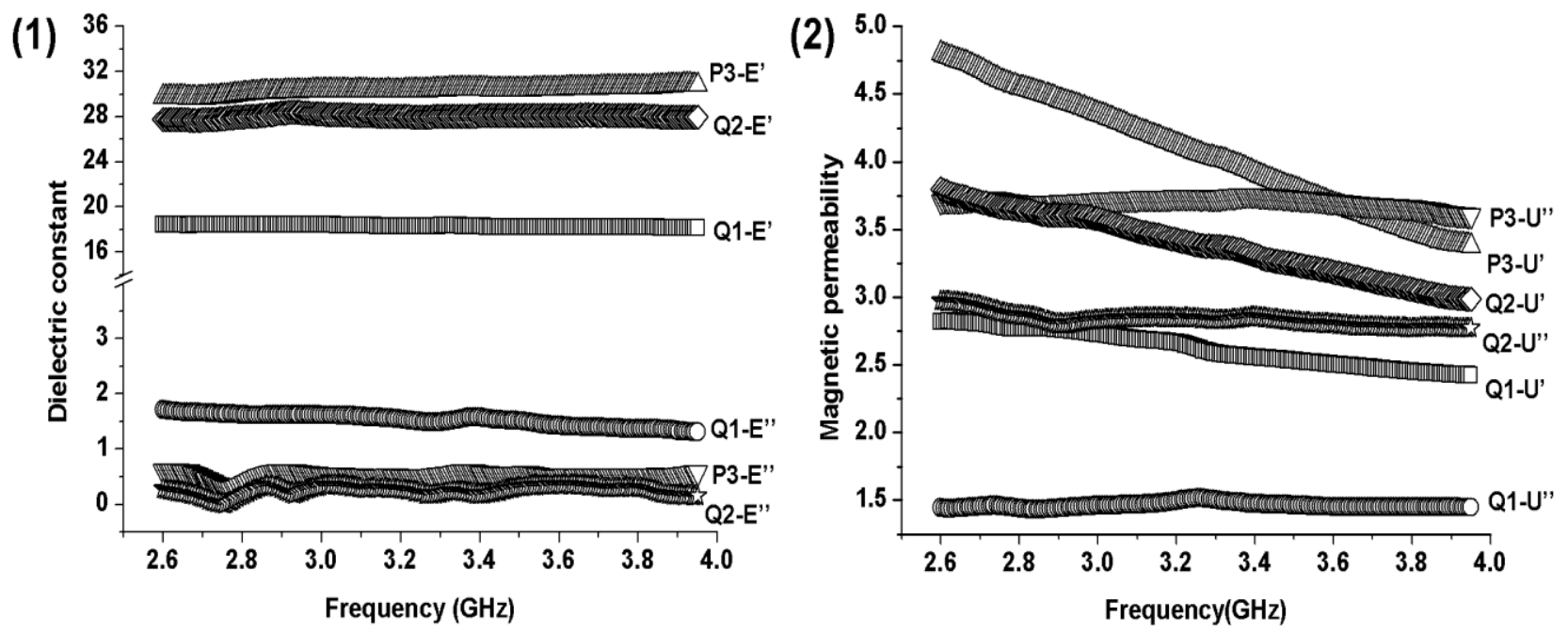

Fig.2. The real parts (E', U') and imaginary parts (E', U') of the relative complex permittivity (1) and permeability (2) for Q1, Q2, and $\mathrm{P} 3$ samples in the range of $2.6-3.95 \mathrm{GHz}$.

Table 2. Electromagnetic (3 GHz) and mechanical properties of Q1, Q2, P3 samples

\begin{tabular}{cccccc}
\hline Materials & $\begin{array}{c}\text { Dielectric } \\
\text { loss }\end{array}$ & $\begin{array}{c}\text { Magnetic } \\
\text { loss }\end{array}$ & $\begin{array}{c}\text { Attenuation } \\
\text { constant } \\
(\mathbf{d B} / \mathbf{c m})\end{array}$ & $\begin{array}{c}\text { Tensile } \\
\text { strength } \\
\mathbf{( M P a})\end{array}$ & $\begin{array}{c}\text { Flexural } \\
\text { strength } \\
\text { (MPa) }\end{array}$ \\
Q1 & 0.088 & 0.535 & 11.772 & 58.74 & 102.6 \\
Q2 & 0.012 & 0.792 & 20.707 & 51.39 & 93.3 \\
P3 & 0.018 & 0.852 & 25.443 & 65.42 & 118.4 \\
\hline
\end{tabular}

\section{Conclusion}

The flaky structure of CIP/epoxy resins composites having excellent electromagnetic property and mechanical performance were successfully prepared using autoclave moulding. According to complex permittivity and permeability, plate-like CIP/epoxy resins composites exhibit better magnetic loss and attenuation constant than spherical CIP/epoxy resins composites at 3 $\mathrm{GHz}$. The improvement of mechanical performance mainly originates from the great contributions of homogenous and dense structure.

\section{References}

1. Adam J D, Davis L E, Dionne G F, et a1. Ferrite devices and materials. IEEE Trans. 50 (3) (2002) 721-737.
2. S.H. Hosseini, A. Asadnia, M. Moloudi, Preparation and electromagnetic wave absorption hard-soft $\mathrm{Ba}$ ferrite/polypyrrole core-shell nanocomposites, Mater. Res. Innovat. 19 (2015) 107-112.

3. Y. Zou, et al., Enhancing and tuning absorption properties of microwave absorbing materials using metamaterials, Appl. Phys. Lett. 93 (26) (2008) 155-160.

4. X. Huang, et al., Tunable electromagnetic properties and enhanced microwave absorption ability of flaky graphite/cobalt zinc ferrite composites, J. Alloys Compounds. 662 (2016) 409-414.

5. R. Panwar, V. Agarwala, D. Singh, A cost effective solution for development of broadband radar absorbing material using electronic waste, Ceram. Int. 41 (2) (2015) 2923-2930. 